\title{
Effects of thymectomy on late-onset non-thymomatous myasthenia gravis: systematic review and meta-analysis
}

\author{
Jinwei Zhang, Yuan Chen, Hui Zhang, Zhaoyu Yang and Peng Zhang * (1)
}

\begin{abstract}
Background: The effects of thymectomy on late-onset non-thymomatous myasthenia gravis (NTMG) remain controversial. The objective of this study was to conduct a systematic review in order to answer two questions pertinent to late-onset NTMG: (1) do patients with late-onset NTMG experience the same effects from thymectomy as their earlyonset counterparts? (2) Compared with conservative treatment, does thymectomy have any benefits for late-onset NTMG patients?

Methods: We searched the PubMed, EMBASE, and Cochrane Library databases for studies published from January 1 , 1950 to March 10, 2021. Outcomes were measured via clinical stable remission/pharmacological remission (CSR/PR) and improvement rates. We used Stata software to analyze the data.

Results: We ultimately included a total of 12 observational articles representing the best evidence answering the questions of our study objective. Of these, nine studies, which included 896 patients overall (766 early-onset and 230 late-onset), compared postoperative outcomes between early- and late-onset NTMG. The remaining three articles, which included 216 patients (75 in the thymectomy group and 141 in the conservative-treatment group), compared thymectomy with conservative treatment for late-onset NTMG. The early- versus late-onset NTMG studies demonstrated that patients in the former category were $1.95 \times$ likelier than their late-onset counterparts to achieve clinical remission (odds ratio [OR] 1.95; 95\% confidence interval [CI] 1.39-2.73; $P^{2}=0 \%$ ). No difference was seen in improvement or remission + improvement rates between these two groups. When comparing thymectomy with conservative treatments in late-onset NTMG patients, neither did we observe any difference in CSR/PR.

Conclusion: We found that late-onset NTMG patients had a lower chance of achieving CSR after thymectomy than early-onset patients. Thymectomy in late-onset NTMG also yielded no benefit to CSR or PR compared with conservative treatments. In late-onset NTMG patients, thymectomy should therefore be performed with caution, and the appropriate cutoff between early- and late-onset MG should be further explored in order to tailor and execute the proper therapeutic strategies.
\end{abstract}

Keywords: Myasthenia gravis, Early onset, Late onset, Thymectomy, Remission, Systematic review

*Correspondence: zhang_peng1225@sina.com Department of Cardiothoracic Surgery, Tianjin Medical University General Hospital, No.154, Anshan Road, Tianjin, China

\begin{abstract}
Background
Myasthenia gravis (MG) is an autoimmune disease characterized by fatigable weakness of cranial and skeletal muscles with elevated titers of acetylcholine receptor (AChR), muscle-specific receptor tyrosine kinase (MuSK) autoantibodies, or other AChR-related proteins that affect the postsynaptic membrane at the
\end{abstract} original author(s) and the source, provide a link to the Creative Commons licence, and indicate if changes were made. The images or other third party material in this article are included in the article's Creative Commons licence, unless indicated otherwise in a credit line to the material. If material is not included in the article's Creative Commons licence and your intended use is not permitted by statutory regulation or exceeds the permitted use, you will need to obtain permission directly from the copyright holder. To view a copy of this licence, visit http://creativecommons.org/licenses/by/4.0/. The Creative Commons Public Domain Dedication waiver (http://creativeco mmons.org/publicdomain/zero/1.0/) applies to the data made available in this article, unless otherwise stated in a credit line to the data. 
neuromuscular junction [1]. The incidence rate of MG varies with age, gender, ethnicity, thymic histology, clinical presentation, and muscular autoantibodies [2]. The estimated range of incidence is $0.3-2.8$ per 100,000 worldwide, and the median global estimated prevalence is 10 per 100,000 [3]. In China, analysis across age groups shows that the incidence of MG increases steadily with age over the first seven decades of life, peaking in the $70-74$ years age group (1.89 per $100,000)$ in adults [4].

According to the age at which the first symptom manifests, MG can be divided into early-onset and late-onset subgroups. The cutoff age is usually recommended as $40-50$ years, less often $60-65$ years [5, 6]. The two groups can differ in sex ratio, thymic histology, autoantibody titers, and reaction to thymectomy [7, 8]. Thymectomy in non-thymomatous MG (NTMG) patients has mostly been conducted in earlyonset patients. Current guidelines and consensus statements for patients with early-onset NTMG note that these patients most often have thymic hyperplasia and advise that removal of the hyperplastic thymus might contribute to decreased antibody production $[8$, 9].

Some controversy surrounds the effect of thymectomy in late-onset NTMG patients. The landmark MGTX trial [10] was a multicenter, randomized, raterblinded study that compared the effects of thymectomy + prednisone with those of prednisone alone in NTMG patients with generalized AChR-MG over a 3-year period. Patients who underwent thymectomy had a lower time-weighted average Quantitative Myasthenia Gravis (QMG) score and time-weighted average alternate-day prednisone dose. In addition, the results favored thymectomy for patients who took azathioprine or were hospitalized for MG exacerbations. However, the MGTX trial did not definitively establish the role of thymectomy in late-onset NTMG, nor did it find a significant difference in prednisone dosage or QMG score between the two treatment groups in patients age $\geq 50$ years [10]. Similar results have been observed in other studies [11, 12], though the results thereof have suggested a potential benefit of thymectomy in late-onset NTMG [13-15]. Given the contradictory evidence on the effects of thymectomy, we conducted this systematic review and meta-analysis with the aim of answering two questions pertaining to late-onset NTMG: (1) Compared with early-onset NTMG patients, do late-onset patients experience any effects from thymectomy? (2) Compared with standard conservative treatment, does thymectomy have any benefits in late-onset NTMG patients?

\section{Materials and methods Data sources}

We searched the electronic databases PubMed, EMBASE, and the Cochrane Library for studies published from January 1, 1950 to March 10, 2021. The MeSH terms, EMTREE terms, and keywords used were 'myasthenia gravis' AND 'thymectomy' AND 'remission or improved or improvement' AND 'aged or elder or older or elderly or late-onset or non-thymomatous'. With no restrictions on language or publication status. For non-English articles needing a full-text review, we used translation software to perform detailed evaluations. Every publication, regardless of article type, was carefully screened for eligibility. We then further screened the references of relevant publications that we found in our search for eligible studies.

\section{Study selection}

Two independent reviewers assessed article eligibility; disagreements were resolved through subsequent discussion. Studies were included if they met the following inclusion criteria. (1) The study cohort consisted of NTMG patients who received thymectomy, regardless of surgical method. (2) Outcome comparisons were measured by remission and/or improvement. Remission was defined as complete stable remission (CSR) or pharmacological remission (PR) according to the criteria of the Myasthenia Gravis Foundation of America (MGFA) [16]. Studies using similar definitions of remission were also included; in these cases, we compared these definitions to decrease selection bias. (3) Comparison data could be extracted from the study; i.e., data on thymectomy versus conservative treatment (anticholinesterase, corticosteroids, or immunosuppressants administered either alone or in combination) in late-onset NTMG patients, or early-onset versus late-onset NTMG patients after thymectomy. (4) The cutoff age range was 40-60 years.

Studies were excluded if they met the following criteria: (1) Children/juvenile MG only; (2) ocular MG only; or (3) refractory MG or MG crisis only.

\section{Data extraction and quality assessment}

Two authors independently extracted data from eligible studies and summarized them using a data extraction form. For randomized controlled trials (RCTs), methodological quality was assessed using the quantitative five-point Jadad scale [17]; quality was considered high if Jadad score $>3$. In addition, we used the NewcastleOttawa Scale (NOS) to assess observational studies [18]. We considered scores of $\geq 7$ points to indicate high methodological quality. 


\section{Data synthesis}

Data analyses were performed using Stata statistical software version 15 (StataCorp., College Station, TX, USA). If $I^{2}>0$, we applied a random-effects model. Substantial heterogeneity was considered present when $I^{2}>75 \%$; in these cases, the combination was considered inappropriate, and the results were presented in narrative form. We also assessed the probability of publication bias using Peters' regression test [19]. Statistical significance was defined as two-tailed $P<0.05$.

\section{Results}

\section{Search results and study characteristics}

In our electronic data search we identified 378 articles in PubMed, 614 in EMBASE, and 19 in the Cochrane Library. After excluding duplicates and analyzing titles and abstracts, we selected 100 articles, of which we obtained full paper copies. From these, we ultimately selected a final 12 publications. Nine studies compared outcomes between early- and late-onset NTMG patients after thymectomy [12, 20-27], while three compared the effects of thymectomy with those of conservative treatment on late-onset NTMG [11, 13, 15] (Fig. 1). All studies were observational. The main characteristics and quality assessments of the selected studies are summarized in Tables 1 and 2.

\section{Descriptive analysis of studies}

Studies comparing outcomes of early-onset versus late-onset NTMG patients after thymectomy $(n=9)$

1. Liu et al. [20], at a single center in China, retrospectively reviewed 103 consecutive patients who received thymectomy for NTMG. Median duration of follow-up was 5.2 years. Overall, 48 patients achieved CSR, and 1-, 2-, 3-, 4-, 5-, and 6-year CSR rates were $9 \%, 25 \%, 40 \%, 45 \%, 52 \%$, and $56 \%$, respectively. Univariate analysis of age did not correlate significantly with CSR. With the cutoff age at 40 years, $47 \%$ (27/57) of early-onset and $46 \%(21 / 46)$ of lateonset patients achieved CSR 5 years after surgery. CSR was defined by the MGFA standard.

2. Lin et al. [12], at a single center in Taiwan, retrospectively studied postoperative outcomes of 60 NTMG patients, following up with them for a mean of 44 months. Age $<40$ years was one of the three

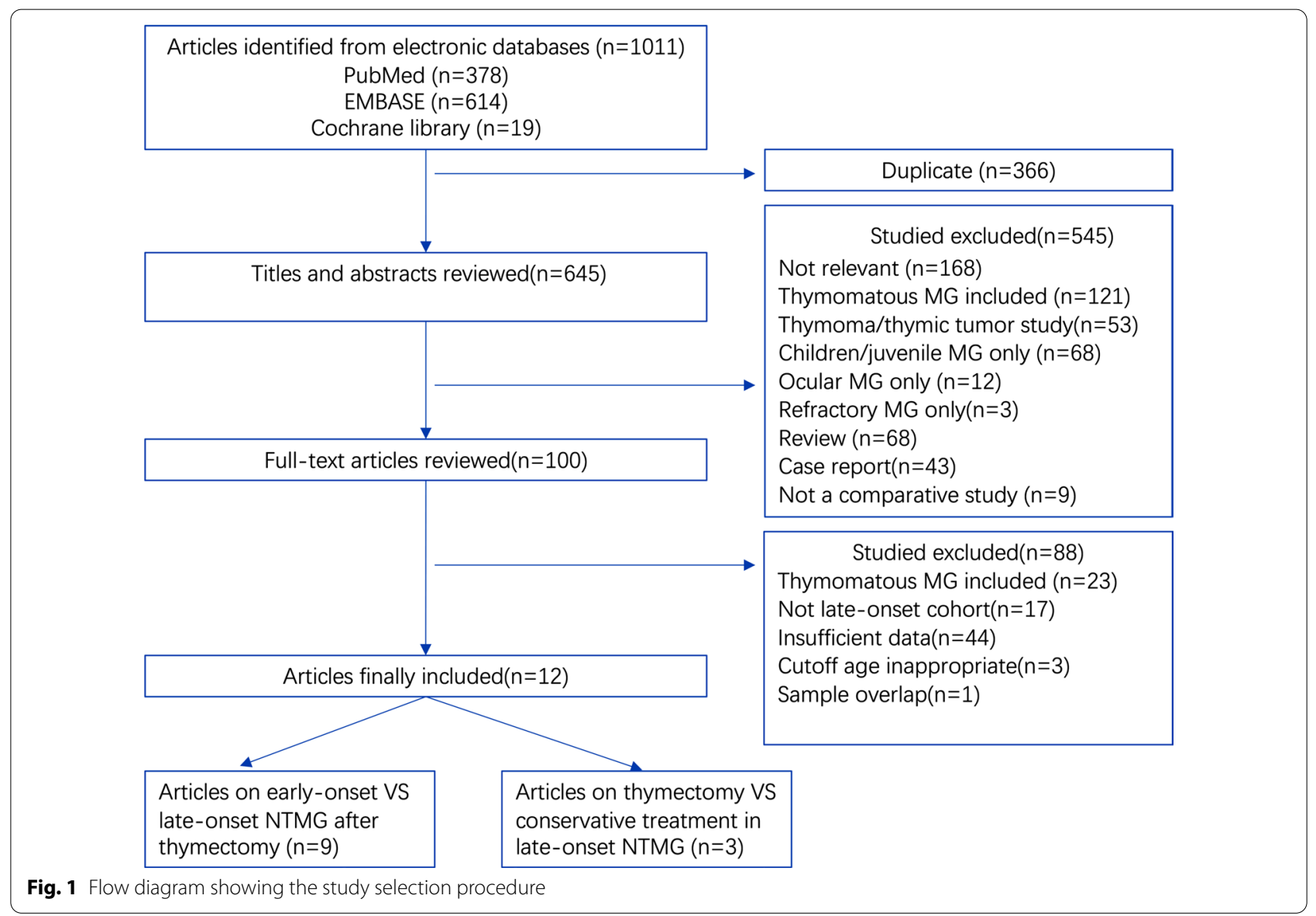




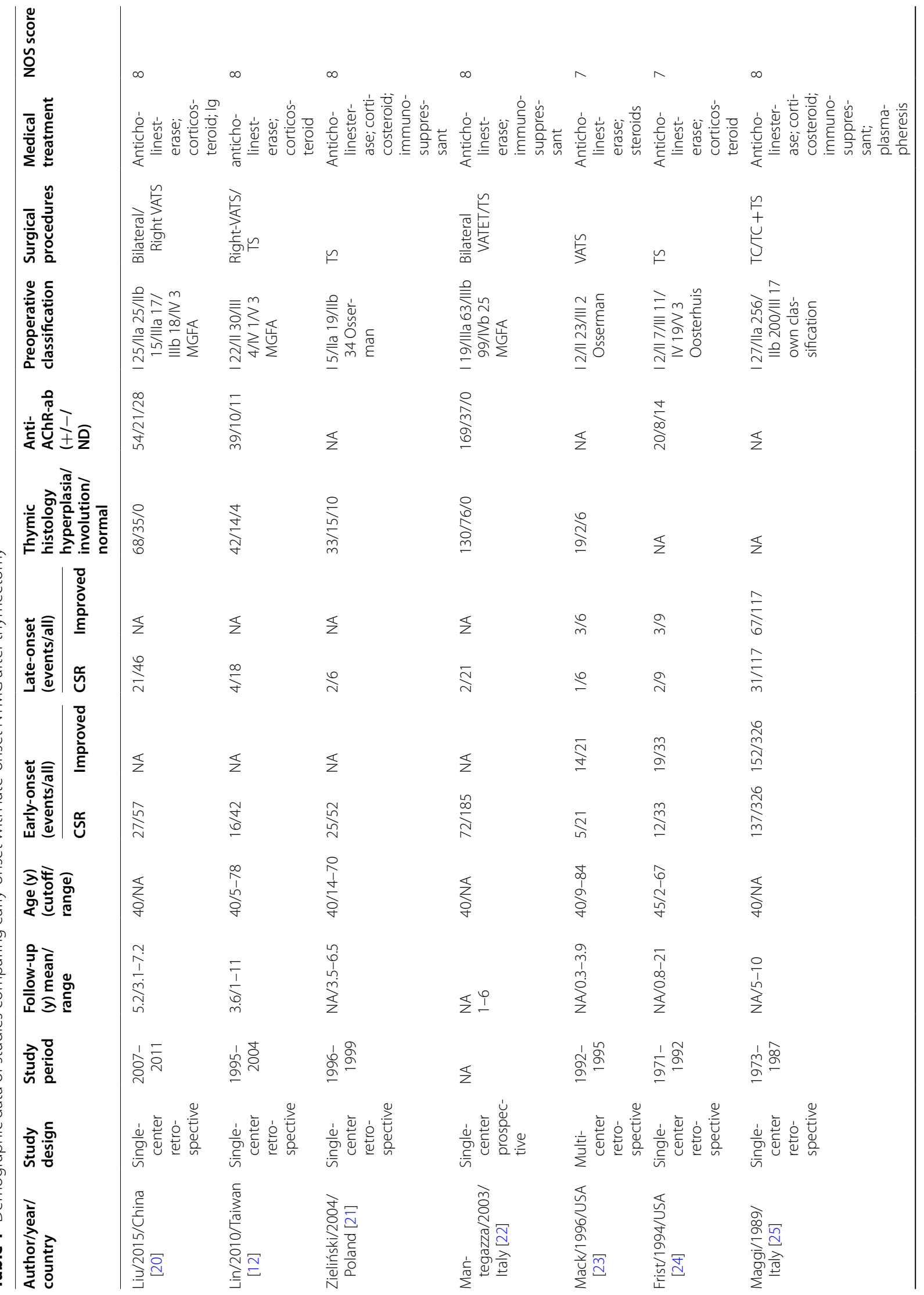




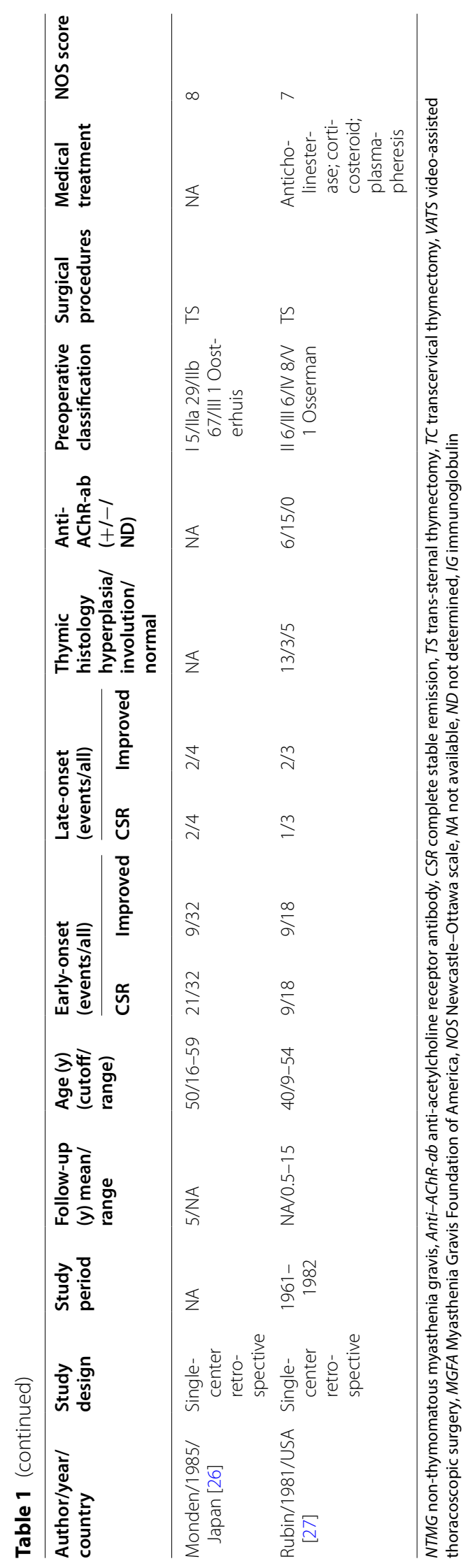


prognostic factors associated with better remission rate. Patients achieving CSR were 38\% (16/42) in the $<40$ years onset age group, compared with $22 \%$ $(4 / 18)$ in the $>40$ years onset age group. CSR was defined by the Clinical Research Standards of the MGFA.

3. Zieliński et al. [21], in a single-center retrospective study in Poland, compared the late results of basic and extended trans-sternal thymectomies. Mean follow-up was 6 years in the basic-thymectomy group and 4 years in the extended-thymectomy group. The complete-remission (CR) rate in the extended group was statistically higher than that in the basic group. However, age ( $<40$ or $>40$ years) had no effect on CR or rates of negative results in either group. Considering that extended thymectomy is already widely recommended as standard procedure [28], we extracted age data from the extended group. We found that $48.1 \%(25 / 52)$ of patients in the early-onset subgroup and $33.3 \%(2 / 6)$ of those in the late-onset subgroup achieved CSR. CR was defined as no symptoms of MG and no need for antimyasthenic medication.

4. Mantegazza et al. [22], in a prospective nonrandomized single-center study in Italy, evaluated 206 NTMG patients for 1-6 years after video-assisted thoracoscopic extended thymectomy (VATET) or trans-sternal thymectomy. Age $<40$ years at onset, thymic hyperplasia, and anticholinesterase drug usage were associated with a significantly greater probability of achieving CSR. In the early-onset group, 38.9\% (72/185) of patients achieved CSR, while $9.5 \%(2 / 21)$ did so in the late-onset group. CSR was defined by the MGFA standard.

5. Mack et al. [23], in a multicenter retrospective study in the USA, included 27 NTMG patients who had undergone thymectomies at four institutions by video-assisted thoracoscopic surgery (VATS) between 1992 and 1995, with 40 years as the cutoff age. CR, which was defined as no symptoms and no need for medications, occurred in 5 of 21 patients in the early-onset group versus 1 of 6 in the lateonset group. Follow-up duration for all patients who achieved CR exceeded 1 year, which was in accordance with the MGFA CSR standard, although overall follow-up time was relatively short (4-47 months).

6. Frist et al. [24], at a single center in Tennessee, USA, retrospectively reviewed the clinical courses of 42 NTMG patients treated with thymectomy via median sternotomy, with a follow-up duration of 10 months-21 years. Twelve (36.4\%) patients in younger group and two (22.2\%) in the older group achieved remission; improvement was seen in 19
(57.6\%) and 3 (33.3\%) patients from these groups, respectively. Outcome criteria were graded according to change from preoperative status to time of most recent follow-up, in accordance with Oosterhuis classification [29]: "remission" implied no symptoms and no medication, "much improved" was defined as a decrease of $\geq 2$ stages, and "improved" was defined as a decrease of 1 stage.

7. Maggi et al. [25], at a single center in Italy, retrospectively studied the post-operative outcomes of 500 NTMG patients. Follow-up data were obtained in 443 cases. CR was achieved in $42 \%(137 / 326)$ of patients $<40$ years of age but in only $26.4 \%(31 / 117)$ of patients in the older group, a statistically significant difference. The improvement rate was $46.6 \%$ $(152 / 326)$ and $57.2 \%$ (67/117), respectively, in these groups. "Remission" was defined as total disappearance of symptoms with no need for treatment; "improvement" was defined as total disappearance of symptoms with occasional need for drugs, or mild symptoms with modest drug intake.

8. Monden et al. [26], in a retrospective single-center study in Japan, compared the clinical features and effects of thymectomy between elderly and young NTMG patients with a cutoff age of 50 years. Rates of remission and palliation (remission + improvement) after thymectomy in elderly patients were respectively 0 and $66.7 \%$ at 6 months, 0 and $50 \%$ at 1 year, $50 \%$ and $100 \%$ at 3 years, and $50 \%$ and $100 \%$ at 5 years. The respective rates in young patients were $18.6 \%$ and $88.4 \%$ at 6 months, $28.2 \%$ and $93.6 \%$ at 1 year, $50 \%$ and $98.1 \%$ at 3 years, and $65.6 \%$ and $93.8 \%$ at 5 years. The difference between the two groups was not significant. Evaluation criteria were defined as follows: remission $=$ no symptoms, or minimal residual symptoms without medication, plus a complete return to work; improved =increased activity with the same amount of medication or less.

9. Rubin et al. [27], in a single-center retrospective study in the USA, reviewed 21 NTMG patients treated with thymectomy with up to 15 years of follow-up. Response to thymectomy was graded as follows: $\mathrm{CR}=$ without medication for $\geq 90$ days; improvement $=$ increased activity with the same or decreased amount of medication. With 40 years as the cutoff age, $50 \%$ of patients $(9 / 18)$ achieved CR in the earlyonset group, and the other half achieved improvement. For late-onset patients, CSR and improvement rates were $33.3 \%(1 / 3)$ and $66.7 \%(2 / 3)$, respectively. 
Studies comparing the effects of thymectomy versus conservative treatment for late-onset NTMG $(n=3)$

1. Kim et al. [15], in a retrospective cohort study in South Korea, compared MGFA post-intervention status (MGFA-PIS) in patients with AChR antibody-positive $\left(\mathrm{AChR}-\mathrm{ab}^{+}\right)$generalized $\mathrm{MG}$ who underwent thymectomy at $\geq 50$ years of age versus those who received medical treatment only. Followup duration was 30-132 months. Cumulative incidence of PR and CSR was significantly higher in the thymectomy group than in the medical-treatment group. Of the 34 patients in the thymectomy group, 6 achieved CSR, and 18 achieved PR. In the medical-treatment group, the success rates were 20/105 for CSR and 28/105 for PR. Note that the number of patients in the medical-treatment group who achieved CSR was not directly shown in the original article; we obtained this data by creating KaplanMeier plots between the two groups using Engauge Digitizer software version 12.2.1.

2. Kawaguchi et al. [13], in a multicenter retrospective study in Japan, performed thymectomy on 20 out of 34 patients (59\%) with late-onset (age $>50$ years) NTMG. Mean follow-up duration was 9.6 years. Six (30\%) patients in the thymectomized group and three (21\%) in the non-thymectomized group achieved remission according to the MGFA-PIS standard (no symptoms; MGFA, score 0). The between-group difference was not statistically significant.

3. Romi et al. [11], in a retrospective single-center study in Norway, evaluated whether the presence of titin and/or RyR antibodies interfered with thymectomy outcome in NTMG patients. Follow-up duration was 2-5 years. Four (titin and RyR antibody negative) thymectomized patients achieved full CR, while two achieved PR (immunosuppressive drugs were used). $\mathrm{CR}$ was seen in three non-thymectomized patients (titin antibody positive); four patients achieved PR. Clinical MG remission was defined as the disappearance of all myasthenic symptoms with (PR) or without (CSR) ongoing medication treatment.

\section{Effects of thymectomy in early-onset versus late-onset patients with NTMG (meta-analysis)}

The authors of all nine studies analyzed CSR between their early- and late-onset NTMG participants [12, 20-27], which in total included 766 patients in earlyonset groups and 230 in late-onset groups. We observed a difference between the two (odds ratio [OR] 1.95; 95\% confidence interval [CI] 1.39-2.73; $I^{2}=0 \%$; Fig. 2): the early-onset groups had $1.95 \times$ greater success in achieving CSR than the late-onset groups. No evidence of publication bias was detected by Peters' test $(P=0.571)$.

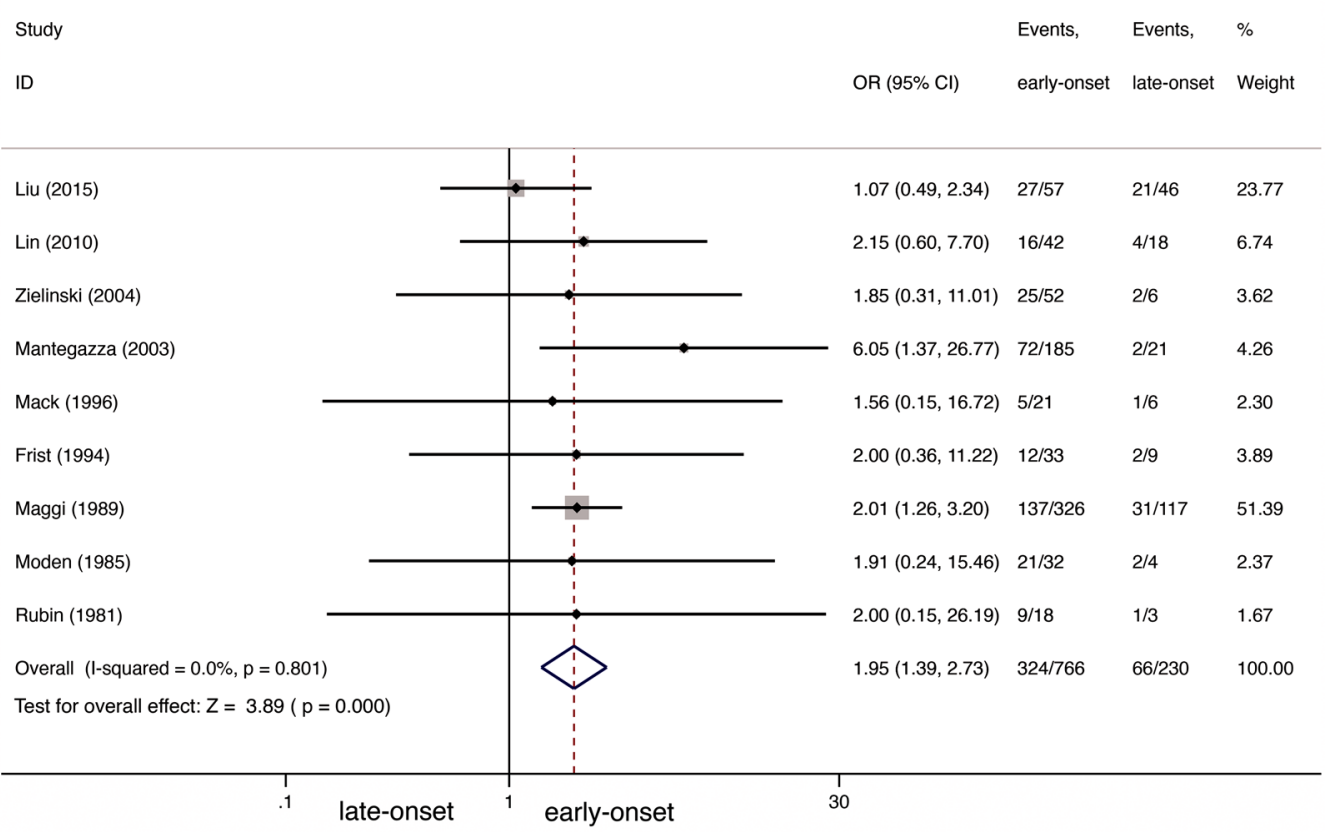

Fig. 2 CSR comparison between early-onset and late-onset NTMG after thymectomy 
Only five studies included data on improvement after thymectomy in early-onset versus late-onset NTMG [23-27], including a total of 430 early-onset and 139 lateonset patients. As shown in Fig. 3, we saw no betweengroup difference in improvement rate (OR 0.82; 95\% CI $0.46-1.45 ; I^{2}=13.2 \%$; Fig. 3$)$. Peters' test detected no evidence of publication bias $(P=0.544)$.
We performed a further analysis on CSR+ improvement between early- and late-onset NTMG patients, observing no significant difference between the groups (OR 2.81; 95\% CI 0.96-8.22; $I^{2}=37.8 \%$; Fig. 4). No evidence of publication bias was detected by Peters' test $(P=0.346)$.

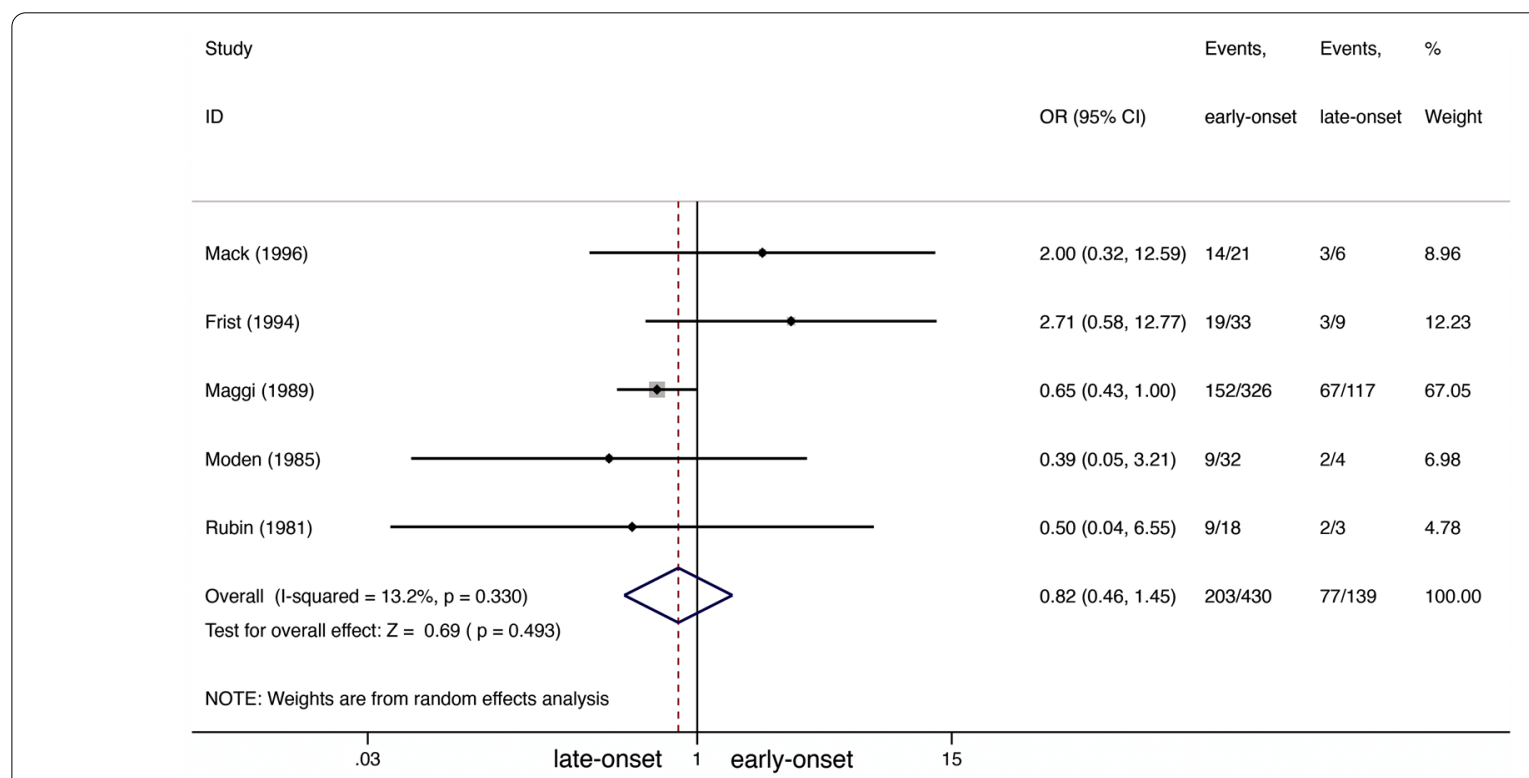

Fig. 3 Improvement comparison between early-onset and late-onset NTMG after thymectomy

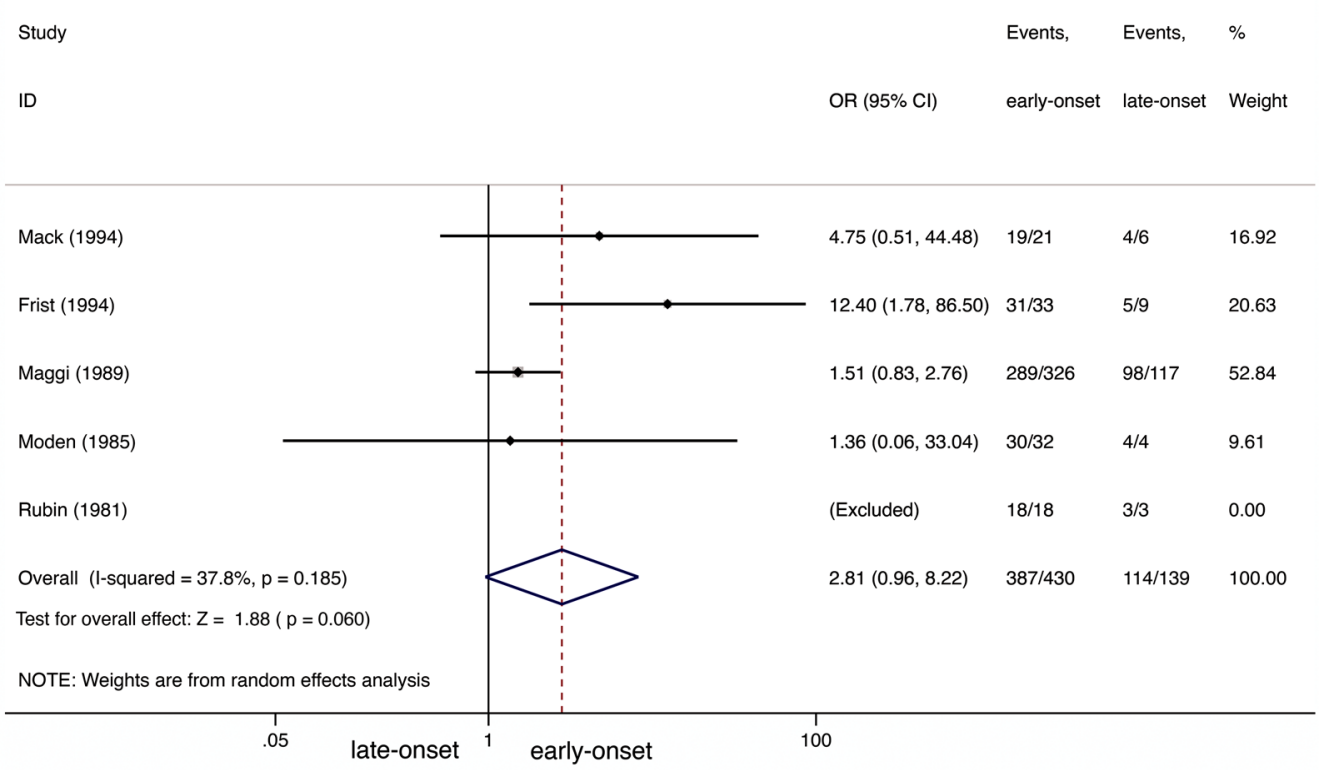

Fig. 4 CSR +improvement comparison between early-onset and late-onset NTMG after thymectomy 


\section{Thymectomy versus conservative treatment in late-onset} NTMG (meta-analysis)

For CSR comparison, due to limited data on thymectomy versus conservative treatment in late-onset NTMG patients, only two studies, including a total of 55 patients in the thymectomy groups and 127 in the conservativetreatment groups, met our criteria [11, 15]. Meta-analysis results did not reveal any difference between thymectomy and conservative treatment for late-onset NTMG
(OR 1.04; 95\% CI 0.45-2.43; $I^{2}=0.0 \%$; Fig. 5). Publication bias was not assessed due to the limited number of studies available.

Similarly, only two studies included PR data on thymectomy versus conservative treatment in NTMG patients $[11,15]$. We observed no statistically significant difference between the two types of treatment (OR 1.45; 95\% CI $0.24-8.87 ; I^{2}=71.1 \%$; Fig. 6). Publication bias was, again, not assessed.

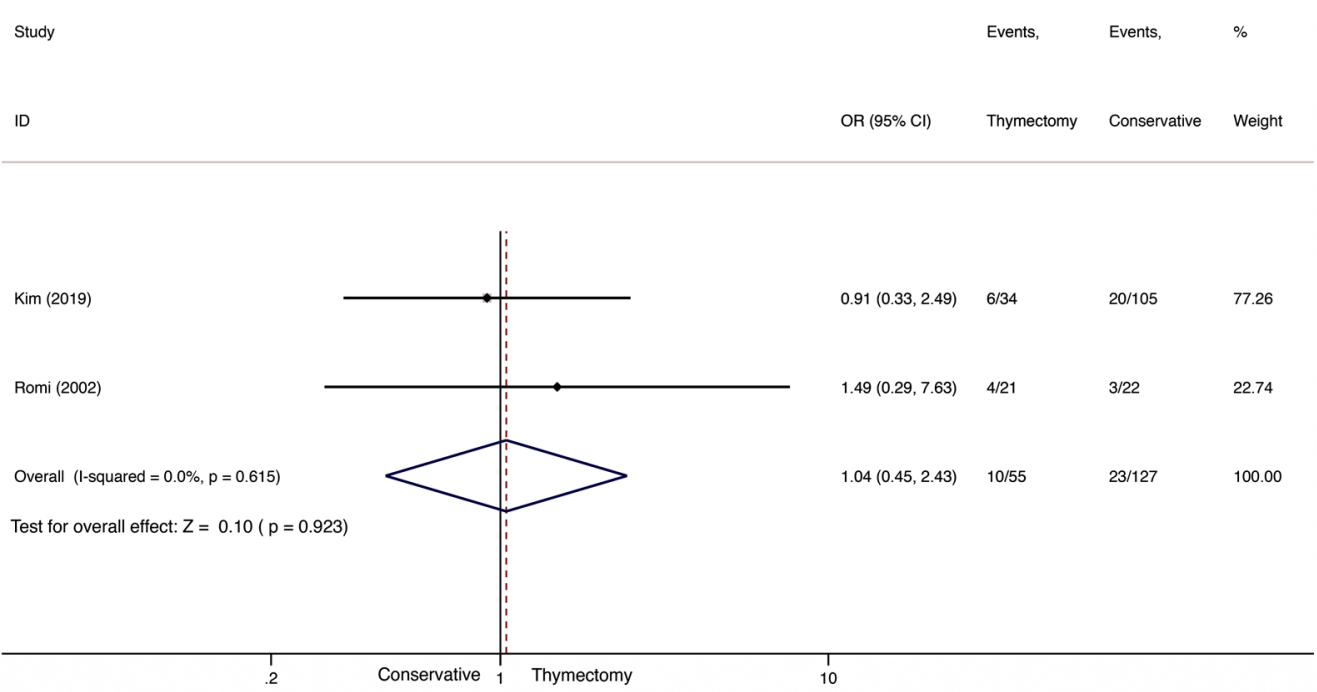

Fig. 5 CSR comparison between thymectomy and conservative treatment in late-onset NTMG

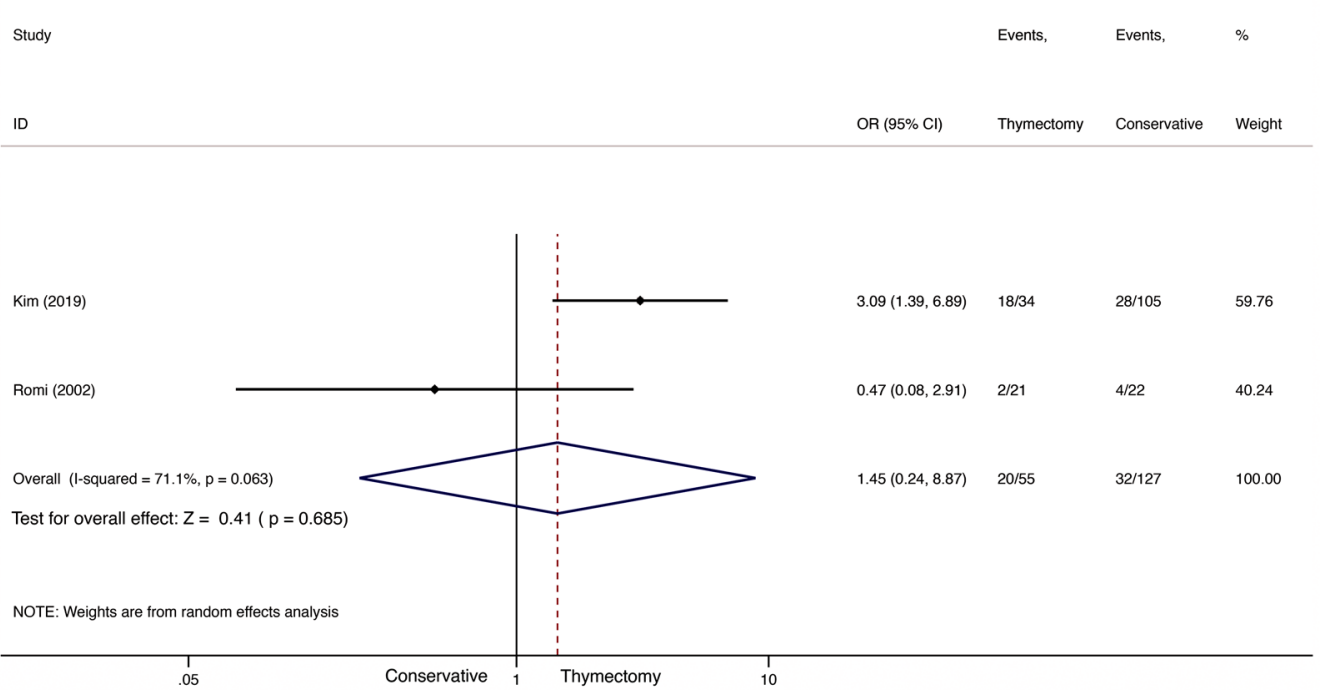

Fig. 6 PR comparison between thymectomy and conservative treatment in late-onset NTMG 


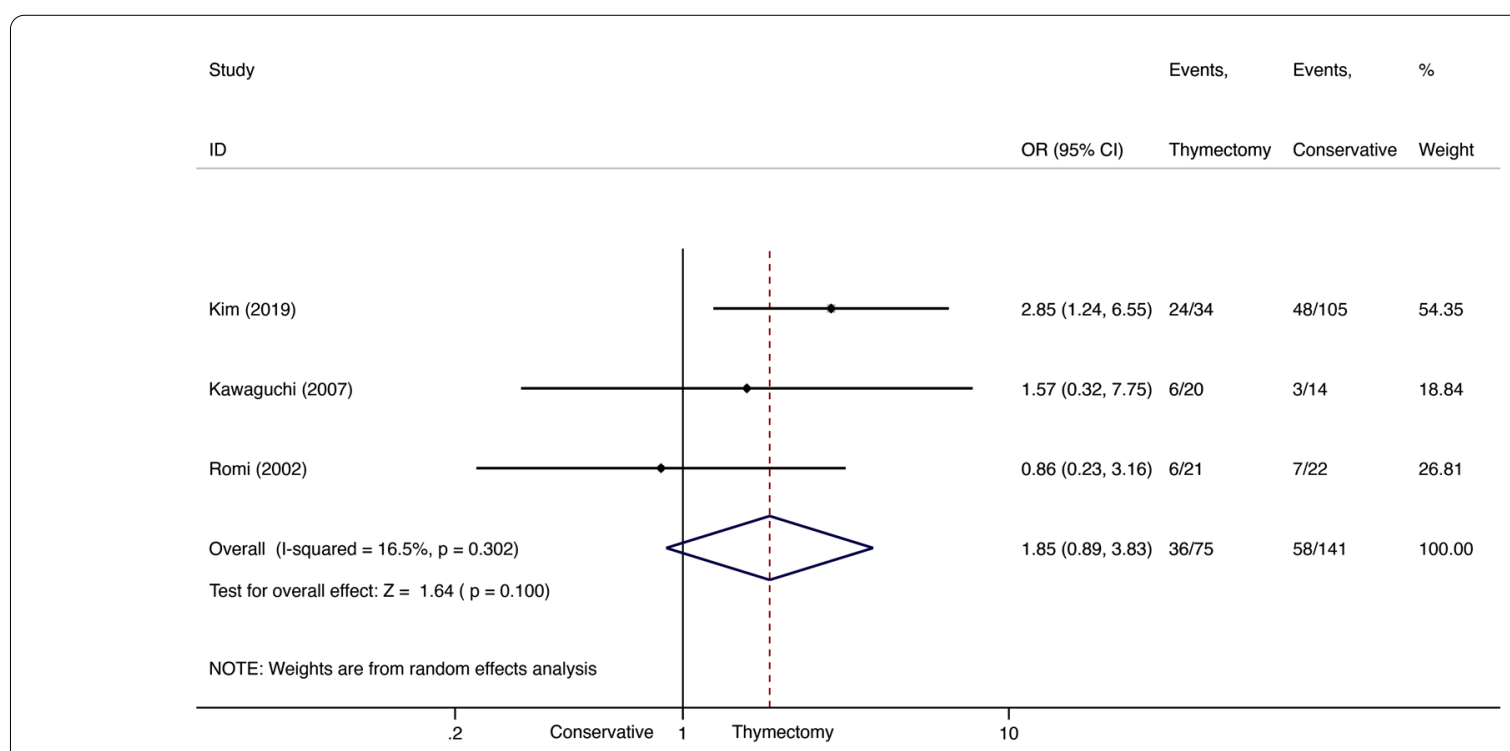

Fig. 7 CSR + PR comparison between thymectomy and conservative treatment in late-onset NTMG

A total of three studies $[11,13,15]$, including a total of 75 patients in the thymectomy groups and 141 in the conservative-treatment groups, were included for CSR + PR comparison. CSR/PR did not differ significantly between groups (OR 1.85; 95\% CI 0.89-3.83; $I^{2}=16.5 \%$; Fig. 7). However, the thymectomy groups achieved CSR/PR at a higher rate than the conservative-treatment groups. We found no evidence of publication bias using Peters' test $(P=0.355)$.

\section{Discussion}

So far, no consensus has been reached on the appropriate cutoff age to divide early-onset from late-onset myasthenia gravis $[5,6,30]$. Many studies have indicated age as one of the factors associated with MG prognosis after thymectomy. However, most of these studies combined thymomatous and non-thymomatous patients in their statistical considerations, potentially confounding the final results. Our work specifically targeted NTMG patients; we extracted nine studies that compared earlywith late-onset NTMG after thymectomy. All of the included studies set a cutoff age between 40 and 50 years. We excluded three studies with cutoff ages of $<40$ years. Of the included studies, three found that early age of onset correlated significantly with better prognosis in NTMG after thymectomy [12, 22, 25], while another three studies found no difference [20, 21, 26]. Furthermore, we found that early-onset patients had a $1.95 \times$ greater CSR rate than late-onset patients. No difference was seen in improvement or CSR+ improvement rates.

While the benefits of thymectomy in NTMG patients have not been conclusively established, there is increasing evidence to support the positive effects thereof compared with medical treatment [31, 32]. Moreover, using high doses of prednisone and/or immunosuppressants has raised some concerns among neurologists. The MGTX study provided cogent evidence that by undergoing thymectomy, generalized NTMG patients who are AChR-ab ${ }^{+}$have a $67 \%$ probability of achieving optimal control of their disease within 12 months. They also benefit from significantly reduced exposure to steroids and immunosuppressants and a much lower rate of treatment-associated symptoms [10]. A meta-analysis by Cataneo et al. also shows the benefits of surgical treatment [33]. However, the MGTX trial did not establish the role of thymectomy in late-onset NTMG patients. Based on existing evidence, a recent International Consensus Guidance (ICG) for MG recommends, "In non-thymomatous, generalized MG patients with AChR-ab, aged $18-50$ years, thymectomy should be considered early in the disease to improve clinical outcomes and to minimize immunotherapy requirements and need for hospitalizations for disease exacerbations [34]."

For NTMG patients age $\geq 50$ years, our database searches retrieved only a few studies that directly compared thymectomy versus conservative treatment in late-onset NTMG. The latest cohort study was a landmark analysis by Kim et al., who found that thymectomy patients had a 2.22-fold better chance of achieving PR than medical-treatment patients after adjusting for age, sex, and disease severity [15]. Kawaguchi et al. [13] conducted a retrospective study with a relatively small number of patients and found no difference in CSR/PR between the thymectomized and conservative groups. 
However, subgroup analyses including 22 patients with mild generalized weakness showed that the thymectomized group had a higher rate of CR and a lower frequency of generalized symptoms than the nonthymectomized group at the end of follow-up, indicating that thymectomy is a potentially effective treatment for NTMG patients with mild generalized MG. Due to selection bias, we could not properly synthesize this subgroup data. However, the study by Romi et al. [11] showed no significant benefit from thymectomy in late-onset NTMG.

Romi et al.'s [11] study also examined whether the therapeutic effect of thymectomy was linked to the presence of non-AChR muscular antibodies. CR or PR was seen in 6 of 11 titin antibody-negative but 0 of the 10 titin antibody-positive thymectomized patients; the non-thymectomized cases showed the opposite trend. A limited number of studies have performed similar analyses. Most researchers have focused on preoperative anti-AChR-ab titers. Among our included studies, three evaluated antiAChR-ab influence CSR in NTMG patients [12, 20, 22]. None of them found evidence to support a correlation between prognosis and these antibodies.

Late-onset MG is often associated with thymic atrophy [7]. Uzawa et al. [35] retrospectively reviewed 2-year post-thymectomy prognoses in 39 consecutive generalized NTMG patients who were AchR-ab ${ }^{+}$(age at onset, $>50$ years). Late-onset NTMG patients with thymic hyperplasia showed a higher rate of remission and received lower prednisolone doses versus patients with involuted thymuses. However, Nakahara et al. compared clinical features and postoperative prognoses among three different thymic histologies and found that the clinical course of MG patients with atrophic thymus after thymectomy was superior to those with hyperplasia or thymoma [36]. Whether thymic histology correlates with response to thymectomy in NTMG is still unclear, but we found significant proportional heterogeneity of thymic histology in our three included studies (Table 2). Histological examination showed that all patients in Romi's study had thymic atrophy [11]. However, Kawaguchi's study [13] provided no thymic-histology data.

Considering how surgical procedures correlate with the effects of surgery in NTMG. Liu et al. [20] compared right-sided (unilateral) with bilateral VATET and found no difference in long-term outcome. Lin et al. [12] compared the results of VATS thymectomy with those of trans-sternal thymectomy and found that the two methods had equivalent CSR rates but the former resulted in shorter hospital stay, less tissue injury, and better cosmetic outcome. In Zielinski et al.'s [21] comparison of $\mathrm{CR}$ rates between basic trans-sternal thymectomy and extended trans-sternal thymectomy, outcomes were considerably better in the extendedthymectomy group. Considering the wide acceptance of extended thymectomy as the current standard procedure [27], we extracted data only from the extended subgroup. Minimally invasive techniques seemed to achieve the same benefits as trans-sternal methods; however, well-designed controlled studies are still needed [28]. Among our included studies, one included a group of patients who received transcervical surgery [25], which can weaken postoperative outcome [28]. Moreover, two studies did not describe the exact surgical procedure $[13,15]$. All three studies therefore presented sources of potential heterogeneity in our analyses.

Preoperative severity may correlate with prognosis for thymectomy in NTMG $[24,25]$. Of the studies on thymectomy versus conservative treatment in late-onset NTMG that we selected, two found no significant difference in terms of preoperative MGFA classification [11, 15]. One study had more severe patients in its thymectomy group than in its nonthymectomy group [13], although final outcomes were not significantly different between the two groups.

To our knowledge, this is the first systematic review and meta-analysis of thymectomy in late-onset NTMG. Despite the small number of observational studies included, our meta-analysis showed a difference between early- and late-onset NTMG patients in terms of CR but not in the effects of thymectomy versus conservative treatment in late-onset NTMG. However, our study had several limitations. First, all studies included were observational, and such studies should be interpreted with caution. Second, the number of studies included was relatively small. Third, the inherent heterogeneity of patient populations and intervention groups could have introduced unknown sources of bias into the results. More multi-center studies with large sample sizes and wellcontrolled cases are needed to limit bias in the future. Moreover, the appropriate cutoff age dividing early- from late-onset MG needs to be further investigated in order to develop tailored therapeutic strategies.

\section{Conclusion}

We observed that late-onset NTMG patients had a lower chance of achieving CSR after thymectomy than early-onset patients, but no difference was seen in improvement or in CSR + improvement rates. Moreover, late-onset NTMG patients did not obtain any benefits from thymectomy versus conservative treatments. Thymectomy in late-onset NTMG patients should therefore be performed with caution, and further investigation into cutoff ages is needed to deliver specific therapeutic strategies. 


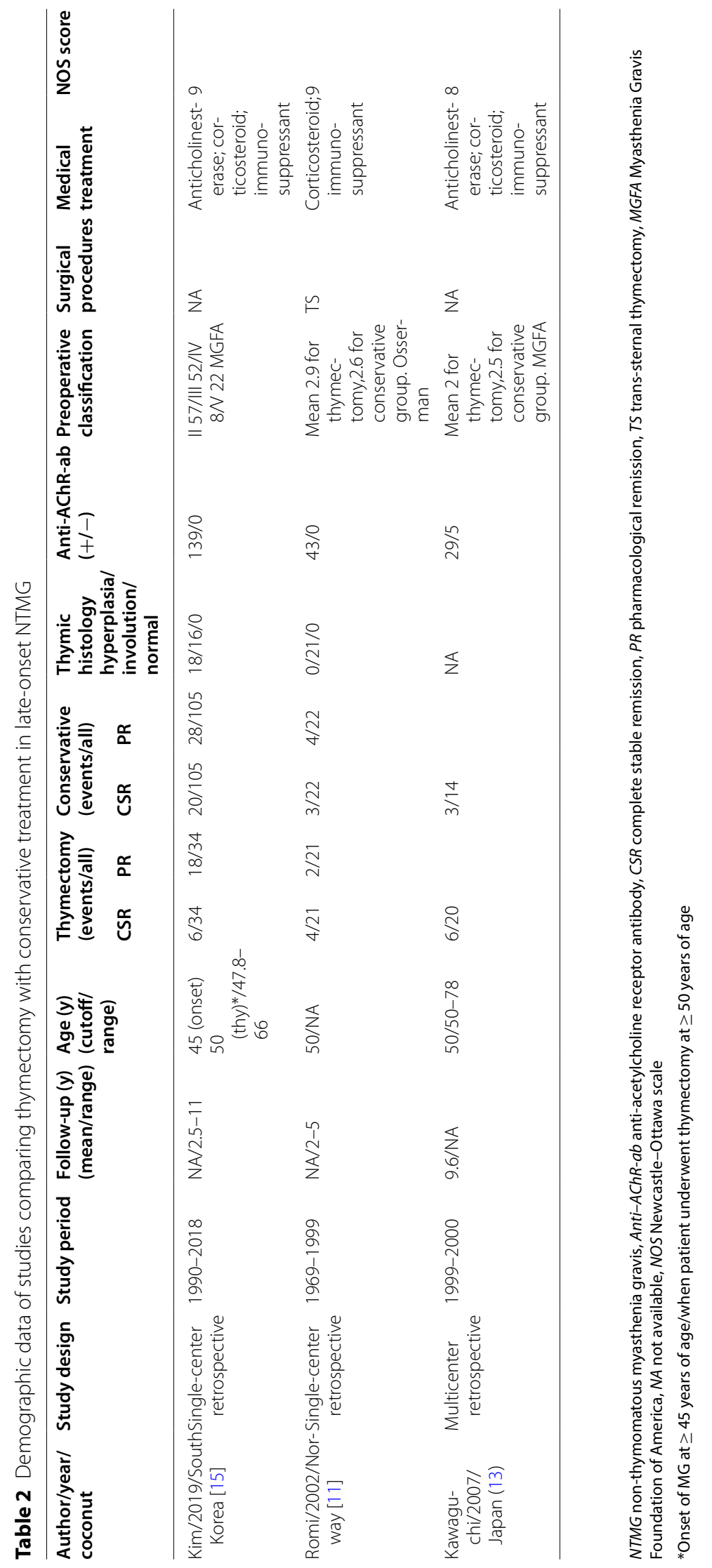




\section{Acknowledgements}

We thank LetPub (www.letpub.com) for its linguistic assistance during the preparation of this manuscript.

\section{Authors' contributions}

JWZ and PZ conceived and designed the study. JWZ and YC selected the articles and extracted the data. $H Z$ and ZYY analyzed the data. JWZ wrote the first draft of the manuscript. PZ, YC, and $\mathrm{HZ}$ contributed to data interpretation and substantial revision of the manuscript. All authors read and approved the final manuscript.

\section{Funding}

None.

\section{Availability of data and materials}

We searched the PubMed, EMBASE, and Cochrane Library electronic databases.

\section{Declarations}

\section{Ethical approval}

This study was approved by the Ethics Committee of the Tianjin Medical University General Hospital (Tianjin, China).

\section{Informed consent}

We were exempted from obtaining informed consent because this was a secondary analysis of previously published studies.

\section{Consent for publication}

Written informed consent for publication was obtained from all participants.

\section{Competing interests}

The authors declare that they have no competing interests.

Received: 13 January 2021 Accepted: 7 May 2021

Published online: 20 May 2021

\section{References}

1. Gilhus NE, Tzartos S, Evoli A, Palace J, Burns TM, Verschuuren J. Myasthenia gravis. Nat Rev Dis Primers. 2019;5(1):30.

2. Meriggioli MN, Sanders DB. Autoimmune myasthenia gravis: emerging clinical and biological heterogeneity. Lancet Neurol. 2009;8(5):475-90.

3. Deenen JC, Horlings CG, Verschuuren JJ, Verbeek AL, van Engelen BG. The epidemiology of neuromuscular disorders: a comprehensive overview of the literature. J Neuromuscul Dis. 2015;2(1):73-85.

4. Chen J, Tian D-C, Zhang C, Li Z, Zhai Y, Xiu Y, et al. Incidence, mortality, and economic burden of myasthenia gravis in China: a nationwide population-based study. Lancet Region Health West Pac. 2020:5:100063

5. Alkhawajah NM, Oger J. Treatment of myasthenia gravis in the aged. Drugs Aging. 2015;32(9):689-97.

6. Aarli JA. Late-onset myasthenia gravis: a changing scene. Arch Neurol. 1999:56(1):25-7.

7. Gilhus NE, Verschuuren JJ. Myasthenia gravis: subgroup classification and therapeutic strategies. Lancet Neurol. 2015;14(10):1023-36.

8. Marx A, Pfister F, Schalke B, Saruhan-Direskeneli G, Melms A, Ströbel P. The different roles of the thymus in the pathogenesis of the various myasthenia gravis subtypes. Autoimmun Rev. 2013;12(9):875-84.

9. Sanders DB, Wolfe GI, Benatar M, Evoli A, Gilhus NE, Illa I, et al. International consensus guidance for management of myasthenia gravis: executive summary. Neurology. 2016;87(4):419-25.

10. Wolfe Gl, Kaminski HJ, Aban IB, Minisman G, Kuo HC, Marx A, et al. Randomized trial of thymectomy in myasthenia gravis. N Engl J Med. 2016;375(6):511-22.

11. Romi F, Gilhus NE, Varhaug JE, Myking A, Skeie GO, Aarli JA. Thymectomy and anti-muscle autoantibodies in late-onset myasthenia gravis. Eur J Neurol. 2002;9(1):55-61.
12. Lin MW, Chang YL, Huang PM, Lee YC. Thymectomy for non-thymomatous myasthenia gravis: a comparison of surgical methods and analysis of prognostic factors. Eur J Cardiothorac Surg. 2010;37(1):7-12.

13. Kawaguchi N, Kuwabara S, Nemoto Y, Fukutake T, Arimura K, Osame M, et al. Effects of thymectomy on late-onset myasthenia gravis without thymoma. Clin Neurol Neurosurg. 2007;109(10):858-61.

14. Uzawa A, Kawaguchi N, Kanai T, Himuro K, Oda F, Yoshida S, et al. Two-year outcome of thymectomy in non-thymomatous late-onset myasthenia gravis. J Neurol. 2015;262:1019-23.

15. Kim SW, Choi YC, Kim SM, Shim HS, Shin HY. Effect of thymectomy in elderly patients with non-thymomatous generalized myasthenia gravis. J Neurol. 2019;266(4):960-8.

16. Jaretzki A III, Barohn RJ, Ernstoff RM, Kaminski HJ, Keesey JC, Penn AS, et al. Myasthenia gravis: recommendations for clinical research standards. Task force of the Medical Scientific Advisory Board of the Myasthenia Gravis Foundation of America. Ann Thorac Surg. 2000;70(1):327-34

17. Jadad AR, Moore RA, Carroll D, Jenkinson C, Reynolds DJ, Gavaghan DJ, et al. Assessing the quality of reports of randomized clinical trials: is blinding necessary? Control Clin Trials. 1996:17(1):1-12.

18. Stang A. Critical evaluation of the Newcastle-Ottawa scale for the assessment of the quality of nonrandomized studies in meta-analyses. Eur J Epidemiol. 2010;25(9):603-5.

19. Peters JL, Sutton AJ, Jones DR, Abrams KR, Rushton L. Comparison of two methods to detect publication bias in meta-analysis. JAMA. 2006;295(6):676-80.

20. Liu Z, Yang J, Lin L, Huang J, Jiang G. Unilateral video-assisted thoracoscopic extended thymectomy offers long-term outcomes equivalent to that of the bilateral approach in the treatment of non-thymomatous myasthenia gravis. Interact Cardiovasc Thorac Surg. 2015;21(5):610-5.

21. Zieliński M, Kuzdzal J, Szlubowski A, Soja J. Comparison of late results of basic transsternal and extended transsternal thymectomies in the treatment of myasthenia gravis. Ann Thorac Surg. 2004;78(1):253-8.

22. Mantegazza R, Baggi F, Bernasconi P, Antozzi C, Confalonieri P, Novellino $\mathrm{L}$, et al. Video-assisted thoracoscopic extended thymectomy and extended transsternal thymectomy (T-3b) in non-thymomatous myasthenia gravis patients: remission after 6 years of follow-up. J Neurol Sci. 2003:212(1-2):31-6.

23. Mack MJ, Landreneau RJ, Yim AP, Hazelrigg SR, Scruggs GR, Kirschner PA, et al. Results of video-assisted thymectomy in patients with myasthenia gravis. J Thorac Cardiovasc Surg. 1996;112(5):1352-60.

24. Frist WH, Thirumalai S, Doehring CB, Merrill WH, Stewart JR, Feniche $\mathrm{GM}$, et al. Thymectomy for the myasthenia gravis patient: Factors influencing outcome. Ann Thorac Surg. 1994;57(2):334-8.

25. Maggi G, Casadio C, Cavallo A, Cianci R, Molinatti M, Ruffini E. Thymectomy in myasthenia gravis. Results of 662 cases operated upon in 15 years. Eur J Cardio-Thorac Surg. 1989;3(6):504-9 (discussion 10-11).

26. Monden $Y$, Nakahara K, Fujii Y. Myasthenia gravis in elderly patients. Ann Thorac Surg. 1985;39(5):433-6.

27. Rubin JW, Ellison RG, Moore HV, Pai GP. Factors affecting response to thymectomy for myasthenia gravis. J Thorac Cardiovasc Surg. 1981;82(5):720-8

28. Gronseth GS, Barohn R, Narayanaswami P. Practice advisory: thymectomy for myasthenia gravis (practice parameter update): report of the Guideline Development, Dissemination, and Implementation Subcommittee of the American Academy of Neurology. Neurology. 2020;94(16):705-9.

29. Oosterhuis HJ. The natural course of myasthenia gravis: a long term follow up study. J Neurol Neurosurg Psychiatry. 1989;52(10):1121-7.

30. Cortés-Vicente E, Álvarez-Velasco R, Segovia S, Paradas C, Casasnovas C, Guerrero-Sola A, et al. Clinical and therapeutic features of myasthenia gravis in adults based on age at onset. Neurology. 2020;94(11):e1171-80.

31. Bachmann K, Burkhardt D, Schreiter I, Kaifi J, Schurr P, Busch C, et al. Thymectomy is more effective than conservative treatment for myasthenia gravis regarding outcome and clinical improvement. Surgery. 2009;145(4):392-8.

32. Barnett C, Katzberg HD, Keshavjee S, Bril V. Thymectomy for nonthymomatous myasthenia gravis: a propensity score matched study. Orphanet J Rare Dis. 2014;9:214. 
33. Cataneo AJM, Felisberto G Jr, Cataneo DC. Thymectomy in nonthymomatous myasthenia gravis-systematic review and meta-analysis. Orphanet J Rare Dis. 2018;13(1):99.

34. Narayanaswami P, Sanders DB, Wolfe G, Benatar M, Cea G, Evoli A, et al. International consensus guidance for management of myasthenia gravis: 2020 update. Neurology. 2020.

35. Uzawa A, Kawaguchi N, Kanai T, Himuro K, Oda F, Yoshida S, et al. Two-year outcome of thymectomy in non-thymomatous late-onset myasthenia gravis. J Neurol. 2015;262(4):1019-23.
36. Nakahara K, Nakane S, Nakajima M, Yamashita S, Mori T, Ando Y. Effect of thymectomy for thymic atrophy in myasthenia gravis: a retrospective study on 93 patients. J Neuroimmunol. 2017;305:182-5.

\section{Publisher's Note}

Springer Nature remains neutral with regard to jurisdictional claims in published maps and institutional affiliations.
Ready to submit your research? Choose BMC and benefit from:

- fast, convenient online submission

- thorough peer review by experienced researchers in your field

- rapid publication on acceptance

- support for research data, including large and complex data types

- gold Open Access which fosters wider collaboration and increased citations

- maximum visibility for your research: over $100 \mathrm{M}$ website views per year

At BMC, research is always in progress.

Learn more biomedcentral.com/submissions 